\title{
Discovery of large-scale diffuse radio emission and of a new galaxy cluster in the surroundings of MACS J0520.7-1328
}

\author{
G. Macario $^{1}$, H. T. Intema ${ }^{2,3}$, C. Ferrari ${ }^{1}$, H. Bourdin ${ }^{4}$, S. Giacintucci ${ }^{5,6}$, T. Venturi ${ }^{7}$, P. Mazzotta ${ }^{4}$, I. Bartalucci ${ }^{4}$, \\ M. Johnston-Hollitt ${ }^{9}$, R. Cassano ${ }^{7}$, D. Dallacasa ${ }^{7,8}$, G. W. Pratt ${ }^{10}$, R. Kale ${ }^{7,8}$, and S. Brown ${ }^{11}$ \\ ${ }^{1}$ Laboratoire Lagrange, UMR 7293, Université de Nice Sophia-Antipolis, CNRS, Observatoire de la Côte d'Azur, 06300 Nice, \\ France \\ e-mail: giulia.macario@oca.eu \\ 2 Jansky Fellow of the National Radio Astronomy Observatory, 520 Edgemont Road, Charlottesville, VA 22903-2475, USA \\ 3 National Radio Astronomy Observatory, PO Box O, 1003 Lopezville Road, Socorro, NM 87801-0387, USA \\ 4 Dipartimento di Fisica, Università degli Studi di Roma “Tor Vergata”, via della Ricerca Scientifica 1, 00133 Roma, Italy \\ 5 Department of Astronomy, University of Maryland, College Park, MD 20742, USA \\ 6 Joint Space-Science Institute, University of Maryland, College Park, MD 20742-2421, USA \\ 7 INAF-Istituto di Radioastronomia, via Gobetti 101, 40129 Bologna, Italy \\ 8 Dipartimento di Fisica e Astronomia, via Ranzani 1, 40127 Bologna, Italy \\ 9 School of Chemical \& Physical Sciences, Victoria University of Wellington, PO Box 600, 6140 Wellington, New Zealand \\ ${ }^{10}$ Laboratoire AIM, IRFU/Service d'Astrophysique CEA/DSM CNRS Université Paris Diderot, Bt. 709, CEA-Saclay, \\ 91191 Gif-sur-Yvette Cedex, France \\ 11 Department of Physics and Astronomy, University of Iowa, 203 Van Allen Hall, Iowa City, IA 52242, USA
}

Received 17 December 2013 / Accepted 17 February 2014

ABSTRACT

\begin{abstract}
We report the discovery of large-scale diffuse radio emission south-east of the galaxy cluster MACS J0520.7-1328, detected through high-sensitivity Giant Metrewave Radio Telescope $323 \mathrm{MHz}$ observations. This emission is dominated by an elongated diffuse radio source and surrounded by other features of lower surface brightness. Patches of these faint sources are marginally detected in a $1.4 \mathrm{GHz}$ image obtained through a re-analysis of archival NVSS data. Interestingly, the elongated radio source coincides with a previously unclassified extended X-ray source. We perform a multi-wavelength analysis based on archival infrared, optical, and X-ray Chandra data. We find that this source is a low-temperature $(\sim 3.6 \mathrm{keV})$ cluster of galaxies, with indications of a disturbed dynamical state, located at a redshift that is consistent with the one of the main galaxy cluster MACS J0520.7-132 $(z=0.336)$. We suggest that the diffuse radio emission is associated to non-thermal components in the intracluster and intergalactic medium in and around the newly detected cluster. We are planning deeper multi-wavelength and multi-frequency radio observations to accurately investigate the dynamical scenario of the two clusters and to address the nature of the complex radio emission more precisely.
\end{abstract}

Key words. galaxies: clusters: individual: MACS J0520.7-1328 - galaxies: clusters: individual: 1WGA J0521.0-1333 radio continuum: galaxies - X-rays: galaxies: clusters

\section{Introduction}

In the currently supported hierarchical scenario of structure formation, galaxy clusters assemble and evolve through mergers and accretion of smaller units of matter at the intersection of cosmic filaments connecting clusters into the large-scale structure of the Universe.

In addition to the most common methods for clusters detections - based on the study of galaxy over-densities, lensing, extended X-ray emission due to thermal bremsstrahlung from the intracluster medium (ICM), and the sub-mm signal due to inverse Compton scattering of cosmic microwave background photons by hot intracluster electrons (SZ effect) - observations in the radio band can also probe for the existence of clusters and/or large-scale structures. Powerful high-redshift radio galaxies are known to be efficient tools for detecting galaxy clusters at high redshifts $(z>1.5$; e.g. Galametz et al. 2013, and reference therein). At lower redshift, tailed radio galaxies are being extensively and successfully used for cluster searches. Since they are known to be associated with galaxy clusters, they can be used to trace the high-density environments in the Universe (e.g. Giacintucci \& Venturi 2009). Several previously unobserved clusters have been actually identified thanks to the detection of tailed sources (e.g. Blanton et al. 2003; Smolčić et al. 2007; Giacintucci \& Venturi 2009; Kantharia et al. 2009; Mao et al. 2010).

Beyond tailed radio galaxies, it is nowadays known that a fraction of merging clusters host diffuse Mpc-scale radio emission (such as radio haloes and relics that are named according with their location and observational properties; e.g. see Ferrari et al. 2008). This synchrotron emission is unrelated to individual cluster radio galaxies and directly probes for non-thermal components (relativistic particles and magnetic fields) mixed with the thermal ICM. To date, all these sources are found to be associated with dynamically disturbed/merging systems (e.g. Cassano et al. 2010b, 2013; Feretti et al. 2012, for a recent review). The detection of such sources can become - in principle - a powerful tool for tracing previously unidentified clusters.

Upcoming deep and wide-field radio surveys with new radio interferometers like Low Frequency Array (LOFAR; e.g. van Haarlem et al. 2013), Australian Square Kilometer Array 
Pathfinder (ASKAP; e.g. Johnston et al. 2008), MeerKAT (e.g. Booth \& Jonas 2012), and Murchison Widefield Array (MWA; e.g. Tingay et al. 2013) will allow systematic searches for new galaxy clusters based on the detection of tailed radio galaxies and diffuse extended emission (see e.g. Enßlin \& Röttgering 2002; Cassano et al. 2010a). For these studies, LOFAR and MWA are particularly suitable, thanks to the very large field of view covered at low radio frequencies that makes them powerful survey telescopes (see e.g. the ongoing all-sky surveys LOFAR MSSS, Heald 2013, and MWA GLEAM, Wayth et al., in prep.).

In this paper we present an example of a new cluster detection made possible thanks to deep low-frequency radio observations. We report the serendipitous discovery with the Giant Metrewave Radio Telescope (GMRT) at $323 \mathrm{MHz}$ of new largescale diffuse radio emission in the proximity of the cluster MACS J0520.7-1328 (M 0520 hereinafter).

M0520 is one of the X-ray brightest clusters of galaxies from the MAssive Cluster Survey (MACS, Ebeling et al. 2010). It is located at $z=0.336$ and has an X-ray luminosity of $L_{\mathrm{r} 500,[0.1-2.4 \mathrm{keV}]}=7.8 \times 10^{44} \mathrm{erg} / \mathrm{s}\left(\right.$ Mantz et al. 2010) ${ }^{1}$. M0520 is classified as a relatively relaxed system on the basis of its morphological properties, i.e. a good alignment between the galaxy and ICM distributions, and quite concentric X-ray surface brightness contours in its central 1.5 Mpc region (morphology code 2 in Ebeling et al. 2010). This cluster is also found to be a lensing system, though no mass estimate has been derived from lensing studies (Horesh et al. 2010). M 0520 is identified in the Planck SZ cluster catalogue as PSZ1 G215.29-26.09, with a mass estimated from the SZ of $M_{500} \sim 6.15 \times 10^{14} M_{\odot}$ (Planck Collaboration XXIX 2014).

For our analysis, we made use of our new GMRT radio observations and of multi-wavelength data available from archives and the literature. The paper is presented as follows. In Sect. 2 we describe the new GMRT observations and data reduction, along with the analysis of the detected emission; in Sect. 3, archival Chandra X-ray data analysis of the cluster is presented; in Sect. 4, we report optical/IR analysis based on available public catalogues. A multi-wavelength comparison is reported in Sect. 5, and the results are discussed in Sect. 6.

The adopted cosmology is $\Lambda \mathrm{CDM}$, with $H_{0}=$ $71 \mathrm{~km} \mathrm{~s}^{-1} \mathrm{Mpc}^{-1}, \Omega_{\mathrm{m}}=0.27, \Omega_{\Lambda}=0.73$. At the redshift of the cluster, $1^{\prime}$ corresponds to $\sim 290 \mathrm{kpc}$.

\section{Radio analysis}

\subsection{Observations and data reduction}

M0520 was observed with the GMRT at $323 \mathrm{MHz}$ in November 2011, as part of an ongoing large observational programme ( $\sim 350 \mathrm{~h}$ of total observing time) named the MACS-Planck Radio Halo Cluster Project. The project is devoted to searching for diffuse radio emission in a sample of galaxy clusters selected from the MACS (X-ray) and the XMM-Planck (X-ray/SZ) catalogues in the redshift range 0.3-0.45, which is relatively unexplored in terms of deep radio observations. For this project, we recently completed a deep pointed survey carried out mainly at low-frequency (25 targets with the GMRT at $323 \mathrm{MHz}, 7$ with the Australia Telescope Compact Array - ATCA at $2.1 \mathrm{GHz}$ ), optimised for detecting of diffuse faint cluster radio emission. Results will be presented in a forthcoming paper (Macario et al., in prep.).

\footnotetext{
1 Corrected for our slightly different choice of cosmological parameters.
}

Data were recorded every 16.1 seconds, with 256 frequency channels covering $32 \mathrm{MHz}$ of bandwidth. Data were reduced using the NRAO Astronomical Image Processing System ${ }^{2}$ (AIPS) package and Source Peeling and Atmospheric Modelling (SPAM) software (Intema et al. 2009). After data editing, the remaining effective bandwidth is $31.2 \mathrm{MHz}$, centred at $323 \mathrm{MHz}$. The total effective time on source is around five hours. The flux scale and the bandpass shape were determined from $\sim 25 \mathrm{~min}$ observation of $3 \mathrm{C} 147$ at the end of observations, adopting a flux density of 52.2 Jy at $323 \mathrm{MHz}$ (following the Perley-Taylor 1999 flux scale ${ }^{3}$ ). The amplitude calibration was applied to the target field data, followed by additional RFI flagging and frequency averaging to 24 channels of $1.3 \mathrm{MHz}$ each. We phase-calibrated the target field with a simple point source model derived from the NVSS (Condon et al. 1998) and WENSS (Rengelink et al. 1997), followed by several rounds of wide-field imaging, CLEAN deconvolution and self-calibration. Only the final self-calibration round included amplitude calibration on a one-minute time scale to correct for antenna gain amplitude variations. Before applying, the gains per antenna were normalized using only the target field scan closest in time to the flux calibrator scan, thereby enforcing the correct flux scale across the whole observation run. SPAM ionospheric calibration and imaging was applied to the data. The average residual amplitude errors are estimated to be $\$ 8 \%$ (e.g. Chandra et al. 2004). For more details on the data reduction procedure, we refer to Macario et al. (2013).

Once the calibration converged, a set of final images were obtained, starting from the full resolution and then progressively applying various parameters on data weighting to enhance the information on the diffuse emission, sampled by the shortest baselines.

\subsection{Detection of diffuse extended radio emission}

Figure 1 (left panel) displays contours of the final full-resolution image at $323 \mathrm{MHz}$ (obtained with uniform weighting of the visibilities), corrected for the primary beam. The resolution is $11.8^{\prime \prime} \times 7.8^{\prime \prime}$, and the average $\mathrm{rms}$ noise level is $\sigma_{323 \mathrm{MHz}}=$ $0.11 \mathrm{mJy} \mathrm{beam}^{-1}$. A $19^{\prime} \times 19^{\prime}$ field is shown, centred about $3^{\prime}$ south-east (SE) of the telescope pointing direction (i.e. the centre of the main cluster). The radio map of the central field of M 0520 is totally dominated by a very bright and slightly extended radio source with a flux density of $\sim 0.5 \mathrm{Jy}$, associated with the brightest cluster galaxy (BCG). Residual emission around it is likely due to deconvolution artefacts caused by the relatively strong emission from the BCG, which limits the dynamic range over the central Mpc of the cluster area.

Very interestingly, a diffuse and elongated low surface brightness radio source is located about $8^{\prime} \mathrm{SE}$ of the cluster centre. It shows a quite regular, nearly linear shape, and it has an extension over $\sim 2.8^{\prime}$ along its major axis. We note that this emission is clearly not due to the artefacts caused by the strong central source, which mostly affect a limited area (radius $\sim 1.5^{\prime}$ ) around the BCG. A magnification of the diffuse source is shown in the right-hand panel of Fig. 1, with the radio contours overlaid on the DSS2 optical red image ${ }^{4}$. Two radio sources, named $\mathrm{S} 1$ and $\mathrm{S} 2$, can be distinguished, and are likely associated with optical counterparts. Their flux densities are $6.4 \pm 0.5 \mathrm{mJy}$ (for the point-like source S1) and $12.0 \pm 1.0 \mathrm{mJy}$ (for the double

\footnotetext{
http://www .aips.nrao .edu

http://www.vla.nrao.edu/astro/calib/manual/

4 The M0520 field is not covered by the SDSS.
} 

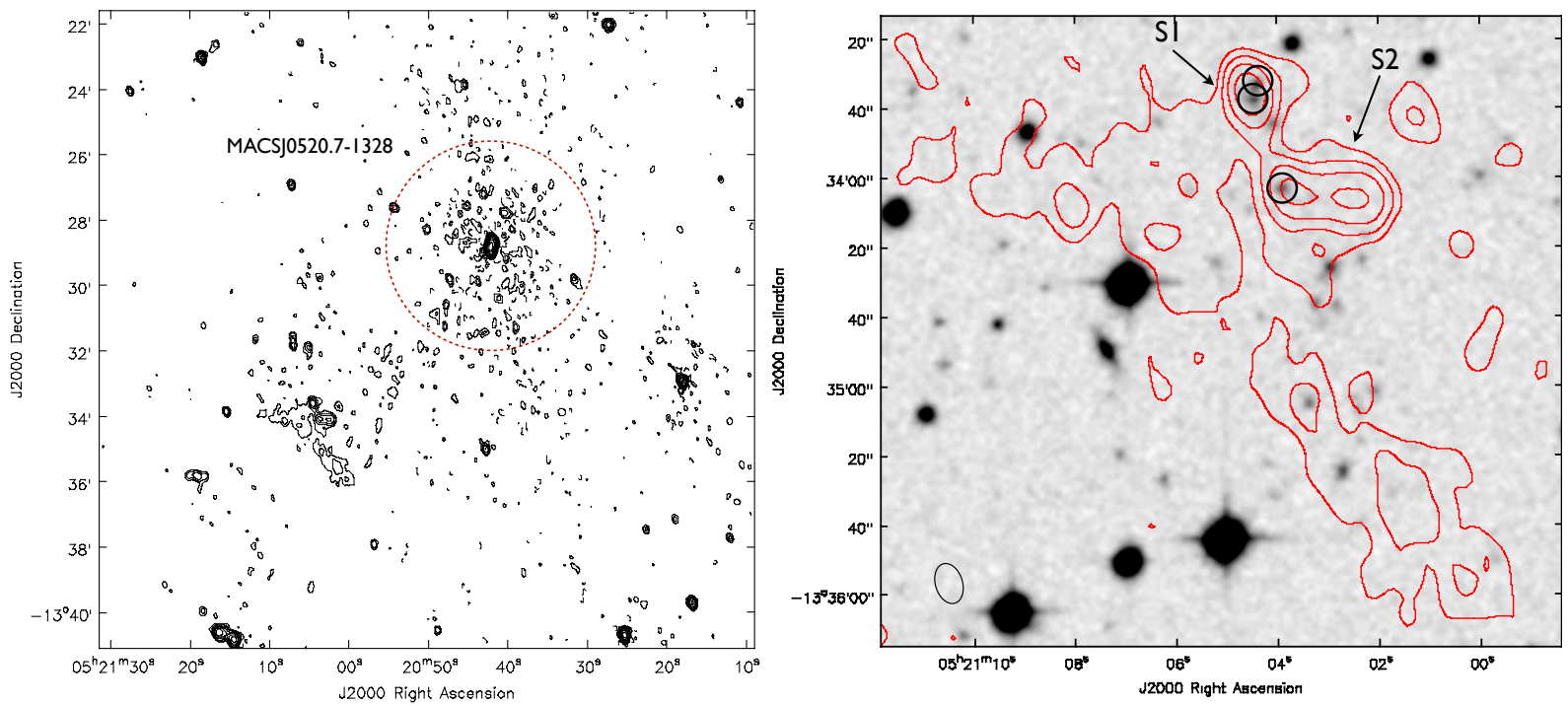

Fig. 1. Left panel: $323 \mathrm{MHz}$ GMRT full-resolution $\left(11.8^{\prime \prime} \times 7.8^{\prime \prime}\right.$, p.a. $\left.16.9^{\circ}\right)$ image of the $19^{\prime} \times 19^{\prime}$ area around the cluster M 0520 . Contours are spaced by a factor of two and start at $\pm 3 \sigma=0.33 \mathrm{mJy} / \mathrm{b}$ (negative contours are dashed). The dashed circle indicates the central region of $1 \mathrm{Mpc}$ radius around the cluster M0520. Right panel: zoom in of the area around the diffuse elongated source; radio contours (same as in left panel) are overlaid on the DSS2 optical red image. The black circles show position of the three galaxies from DSS catalog, which are likely optical counterparts of radio sources $\mathrm{S} 1$ and $\mathrm{S} 2$.
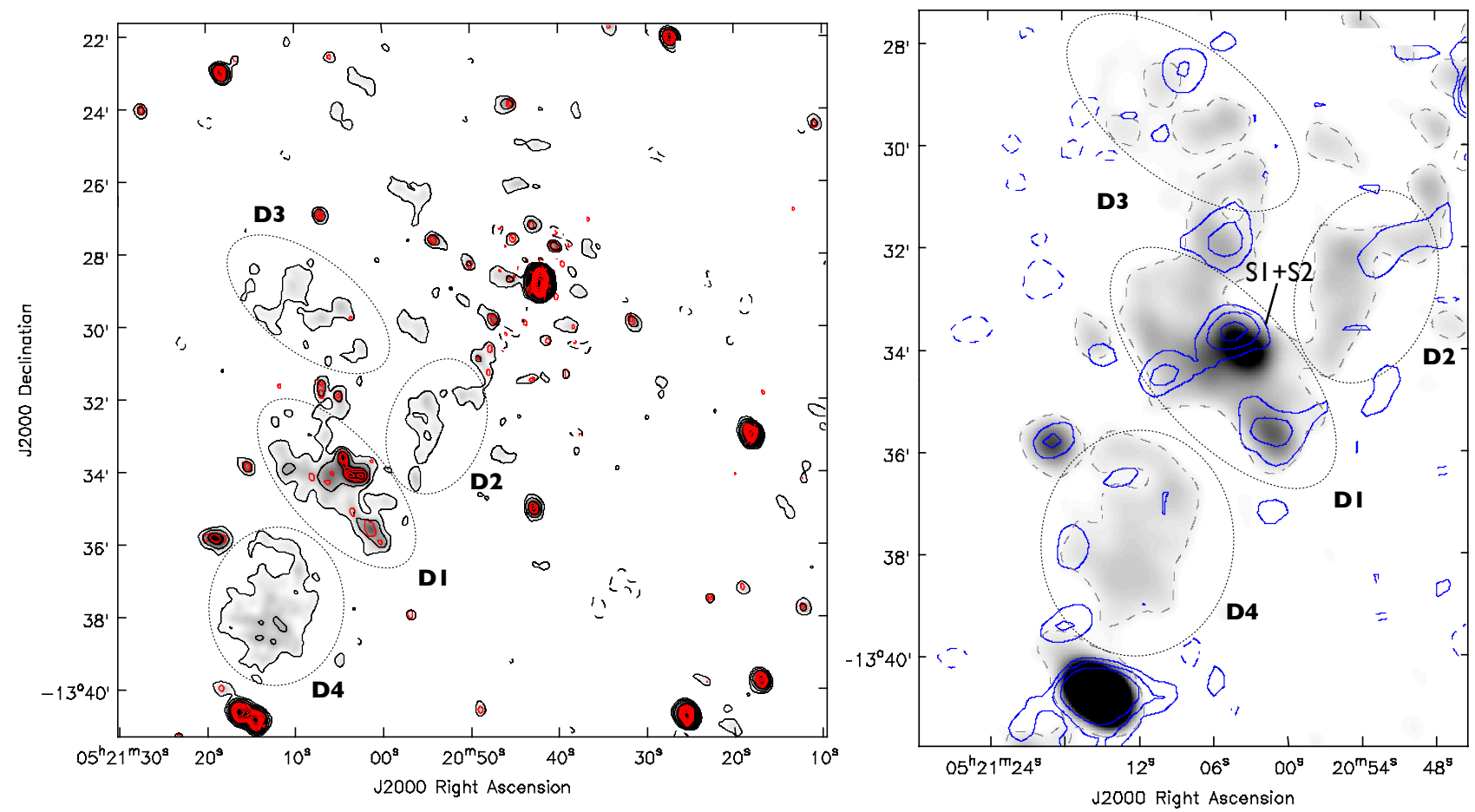

Fig. 2. Left panel: $323 \mathrm{MHz}$ GMRT low-resolution $\left(21.1^{\prime \prime} \times 17.2^{\prime \prime}\right.$, p.a. $\left.28.8^{\circ}\right)$ image of the same $19^{\prime} \times 19^{\prime}$ region around the cluster M $0520($ same as in Fig. 1); black contours are spaced by a factor of two and start at $\pm 3 \sigma_{323 \mathrm{MHz}}=0.82 \mathrm{mJy} / \mathrm{b}$. (Negative contours are dashed.) To highlight the position of discrete compact radio sources in the field, contours from the full-resolution image (see Fig. 1) are over-plotted in red, starting from $\pm 6 \sigma=0.66 \mathrm{mJy} / \mathrm{b}$. Dotted ellipses mark the location of diffuse emission, labelled D1 to D4. Right panel: VLA $1.4 \mathrm{GHz}$ contours (in blue) of an image obtained from re-processed NVSS data $\left(45.8^{\prime \prime} \times 29.9^{\prime \prime}\right.$, p.a. $\left.-70.0^{\circ}\right)$; contours start at $\pm 2 \sigma_{1400 \mathrm{MHz}}=0.5 \mathrm{mJy} / \mathrm{b}$ and are spaced by a factor of 2 (blue dashed contours are negative). The contours are overlaid on a $323 \mathrm{MHz}$ GMRT image of similar resolution $\left(41.5^{\prime \prime} \times 33.5^{\prime \prime}\right.$, p.a. $\left.21.3^{\circ}\right)$, shown in grey scale. (The grey dashed contour corresponds to $3 \sigma_{323 \mathrm{MHz}}=1.65 \mathrm{mJy} / \mathrm{b}$ ).

source S2). The diffuse emission, on the other hand, lacks obvious optical counterparts. We note that a bridge of radio emission seems to connect source S1 with the northern part of the diffuse source.

As Fig. 1 (left panel) shows, peaks of residual emission are spread NW and SE of this elongated source, suggesting the presence of additional underlying extended emission of lower surface brightness. To highlight this feature, as well as to better image the diffuse elongated source, we produced images at lower resolution by using different weighting schemes and by applying Gaussian tapers to give more weight to the short baselines.

In the left-hand panel of Fig. 2 we show a low-resolution image (grey scale and black contours) of the central area around M0520 (same as in Fig. 1, left panel), obtained with robust 0.5 
Table 1. Properties of the diffuse radio emission.

\begin{tabular}{lccccc}
\hline \hline & $\begin{array}{c}\text { Integrated flux densities [mJy] } \\
(323 \mathrm{MHz})\end{array}$ & $\begin{array}{c}\text { Angular extent } \\
(\text { “× } \times)\end{array}$ & $\begin{array}{c}\text { Linear extent } \\
(\mathrm{kpc} \times \mathrm{kpc})\end{array}$ & $\begin{array}{c}P_{323 \mathrm{MHz}^{*}} \\
\left(\mathrm{~W} \mathrm{~Hz}^{-1}\right)\end{array}$ \\
\hline D1 & $61.2 \pm 5.7$ & $9.0 \pm 1.6$ & $2.8 \times 1.4$ & $800 \times 400$ & $2.3 \times 10^{25}$ \\
D2 & $32.0 \pm 3.6$ & $7.2 \pm 1.6$ & $1.8 \times 1.3$ & $520 \times 370$ & $1.2 \times 10^{25}$ \\
D3 & $34.7 \pm 4.0$ & $1.9 \pm 1.4$ & $2.7 \times 1.3$ & $780 \times 375$ & $1.3 \times 10^{25}$ \\
D4 & $48.5 \pm 5.0$ & $8.4 \pm 1.9$ & $2.2 \times 1.9$ & $635 \times 550$ & $1.8 \times 10^{25}$ \\
\hline
\end{tabular}

Notes. ${ }^{(*)}$ Assuming the emission is located at the redshift of M0520.

weighting and a $10 \mathrm{k} \lambda$ Gaussian taper to down-weight long baselines. At this resolution $\left(21.1^{\prime \prime} \times 17.2^{\prime \prime}\right)$, the diffuse elongated source (labelled here as D1) appears slightly more extended, with a more homogeneous brightness distribution. This image also brings forth other patches of diffuse emission surrounding D1, with lower surface brightness and extended on large scales. In particular, NW of D1, a filamentary structure (labelled as D2) extends over $\sim 1.8^{\prime}$ from the SW part of D1 towards the centre of M0520. North of D1 other patches of emission (labelled as D3) are found, with similar orientation as D1 and extended over $\sim 2.7^{\prime}$. A more regular roundish patch $\left(\sim 2^{\prime} \times 2^{\prime}\right)$ of low surface brightness emission (labelled as D4) is located SE of D1.

To check the reliability of this underlying emission (D2, D3, D4), we subtracted from the $u v$-data the contribution from all the unrelated radio sources, including those from D1, and carefully inspected the residual dataset, especially at the short baselines. Neither residual low level RFI nor bad data were found. We imaged the residual dataset with different parameters and found the emission is seen by all baselines shorter than $0.7 \mathrm{k} \lambda$. We also produced images by selecting different groups of channels and time ranges and found the emission is visible at all frequencies and for the whole duration observation. Such emission regions have only positive brightness values, and we can exclude that they are artefacts arising from the bright radio galaxy at the centre of M 0520 (that affect a smaller area around it; i.e. within the dashed circle in Fig. 1). In particular, the extended emission regions D2 and D3 do not have (positive or negative) counterparts at the opposite side of the bright central source, which strongly argues against a connection with its residual sidelobes.

In Fig. 2, the red contours represent the full-resolution image (shown in Fig. 1, left panel), starting at the level of $\pm 6 \sigma$, to help identifying the position of individual discrete radio sources. We note that no discrete radio sources are embedded in the patches of extended emission (D2, D3, D4), excluding any possibility that these features result from the blending of compact sources.

\subsection{Analysis}

To seek for confirmation of the newly detected diffuse emission, as well as to provide a rough estimates of its spectral index, we have inspected all the available images of the M0520 field in the public radio surveys. Owing to their low sensitivities, none of the diffuse components (D1, D2, D3, D4; see Fig. 2, left) are detected either in the $74 \mathrm{MHz}$ VLSS (Kassim et al. 2003; Lane et al. 2008) and VLSSr (Lane et al. 2012) or in the $153 \mathrm{MHz}$ TGSS $^{5}$ surveys.

Hints of diffuse emission are visible in the NVSS (NRAO VLA Sky Survey; Condon et al. 1998) 1.4 GHz image. To improve the quality of the survey image, we have reprocessed

5 http://tgss.ncra.tifr.res.in/150MHz/tgss.html and analysed the NVSS (project AC308) pointing containing the M0520 field. The data were imaged after a new calibration and phase self-calibration in AIPS. The rms sensitivity level achieved in the final image is $\sim 0.25 \mathrm{mJy} /$ beam, with a restoring beam of $45.8^{\prime \prime} \times 29.9^{\prime \prime}$ (p.a. $-70.0^{\circ}$ ), which is about three times lower than the NVSS public image (local noise of $\sim 0.5 \mathrm{mJy} /$ beam, $45^{\prime \prime}$ restoring beam). The residual amplitude errors are $\sim 5 \%$. In the right-hand panel of Fig. 2 we show the contours (in blue) from the final re-calibrated NVSS image (starting from $\pm 2 \sigma_{1400 \mathrm{MHz}}=0.5 \mathrm{mJy} / \mathrm{b}$ level). For a proper comparison, these are overlaid on a $323 \mathrm{MHz}$ GMRT image (in grey scale) of similar resolution $\left(41.5^{\prime \prime} \times 33.5^{\prime \prime}\right)$, obtained using only the visibility data corresponding to baselines shorter than $15 \mathrm{k} \lambda$, with robust 0 weighting and a $4 \mathrm{k} \lambda$ Gaussian taper. The dashed grey contour corresponds to the $+3 \sigma_{323 \mathrm{MHz}}=$ $1.65 \mathrm{mJy} /$ beam level of the image. At the $\sim 2 \sigma_{1400 \mathrm{MHz}}$ level, the diffuse components clearly detected at $323 \mathrm{MHz}$ are only marginally detected in the NVSS data. In particular, when considering the diffuse elongated source D1, only part of the emission SE of S1 and its SW brightest region are detected. Only patches of the emission within the regions D2, D3, and D4 are detected. This is due to the worse surface brightness sensitivity of the NVSS image compared to the $323 \mathrm{MHz}$ image.

We measured the total flux densities of each patch (D1 to D4) at $323 \mathrm{MHz}$ and $1.4 \mathrm{GHz}$ by integrating the two images, corrected for the respective primary beam, over the same areas (i.e. within the dashed ellipses shown in the right-hand panel of Fig. 2). S1 and S2 are blended at the low-resolution of the $1.4 \mathrm{GHz}$ image, not allowing an accurate measurement of their individual flux densities. We thus estimated their global flux density by integrating the two low-resolution images in Fig. 2 (right panel) over a same area that encompasses their emission at both frequencies; we found $\sim 4.5 \mathrm{mJy}$ at $1.4 \mathrm{GHz}$ and $\sim 30 \mathrm{mJy}$ at $323 \mathrm{MHz}$. These values were subtracted from the total flux densities measured in the D1 region to provide an estimate of the flux density of the diffuse emission alone.

The flux density measurements of each component are reported in Table 1, together with their uncertainties. These are computed as

$\sigma_{S}=\sqrt{\left(\sigma_{\mathrm{rms}} \times \sqrt{N_{\text {beam }}}\right)^{2}+\left(\sigma_{\mathrm{amp}}\right)^{2}}$

where $\sigma_{\text {rms }}$ is the rms noise of the image, $N_{\text {beam }}$ the number of independent beams in the region where the flux densities are measured, and $\sigma_{\text {amp }}$ the residual amplitude error.

Owing to the marginal detection in the NVSS, the measurements of the flux densities of each patch of emission at $1.4 \mathrm{GHz}$ are actually affected by large uncertainties. Moreover, part of the flux density is likely missed in the reprocessed NVSS image, due to the very short duration $(\sim 1 \mathrm{~min})$ of the observation, which results in a sparse uv-coverage and loss of sensitivity to the structure. Therefore it is not possible to properly estimate the spectral 
indices for each component. We are planning deeper and higher resolution follow-up observations at $1.4 \mathrm{GHz}$ and other frequencies, which are necessary for a precise spectral analysis and for an accurate study of the newly discovered extended emission.

The angular extent of each component (D1-D4), estimated measuring the major and minor axes of each ellipse of Fig. 2, are reported in Table 1. Under the assumption that those structures are located at the cluster redshift $z=0.336$, their projected linear extents are in the range $500-800 \mathrm{kpc}$ (see Table 1 ). The derived total radio powers at $323 \mathrm{MHz}$ is also reported in Table 1, under the same assumption.

\section{Chandra X-ray analysis}

A simple overlay of the GMRT radio contours on the archival Chandra raw image of M0520 central field $\left(\sim 20^{\prime} \times 20^{\prime}\right)$ immediately revealed that the newly detected diffuse elongated radio source D1 (see Sect. 2.3) coincides with an extended X-ray component, unclassified in the literature. The nature of this diffuse X-ray emission is studied in the following sections through available multi-wavelength data.

\subsection{Data reduction and images}

We re-analysed the Chandra ACIS-I X-ray data from the public archive (obsid: 3272, total exposure $\sim 20 \mathrm{ks}$, which is same as the one analysed in Mantz et al. 2010). A filtering of the hard and soft event light curves only reduced this exposure by a $1 \%$ factor. We binned all photon events in sky coordinates and energy with a fixed angular resolution of $2.5^{\prime \prime}$, and a variable energy resolution matching the camera response. Following an approach described in Bourdin \& Mazzotta (2008), we similarly binned two quantities useful for imaging and spectroscopy: the effective exposure time and the estimate of a background noise level. The effective exposure included the spatially variable mirror effective area and detector quantum efficiency, the CCD gaps and bad pixels, and a correction for the telescope motion. These quantities were extracted from the Chandra Calibration data base (CALDB 4.4.5) and the events list. The background noise model consists of galactic foreground and cosmic X-ray background (CXB) components, but also false detections due to cosmic-rayinduced particles. It has been fit to the data outside the region of the field of view covered by the target. As described in Bartalucci 2014, for each ACIS-I CCD the particle background model holds a spatially variable spectrum including a power law plus exponential continuum and several fluorescence lines. It has been fit to very faint out-of-focus and blank-sky observations performed during the so-called $D$ and $E$ background periods, which cover our observation.

We extracted X-ray images in the $0.5-2.5 \mathrm{keV}$ energy band by correcting the event images for effective exposure and background noise. The image shown in Fig. 3 has been smoothed with a Gaussian of $\sim 5^{\prime \prime}$ FWHM to reduce the shot noise, and covers the same region of the sky as shown in Fig. 1 (left panel). The GMRT $323 \mathrm{MHz}$ contours (same as in Fig. 2, left panel) are overlaid on that.

Beyond the known X-ray emission associated with the galaxy cluster M 0520 (Ebeling et al. 2010; Mantz et al. 2010), we clearly detect a SE diffuse X-ray source, located about $8^{\prime}$ from the centre of the main cluster and coincident with the newly detected diffuse radio source D1. The peak of X-ray emission of this extended structure corresponds to an unclassified source (namely 1WGA J0521.0-1333; hereafter 1WGA 0521) in

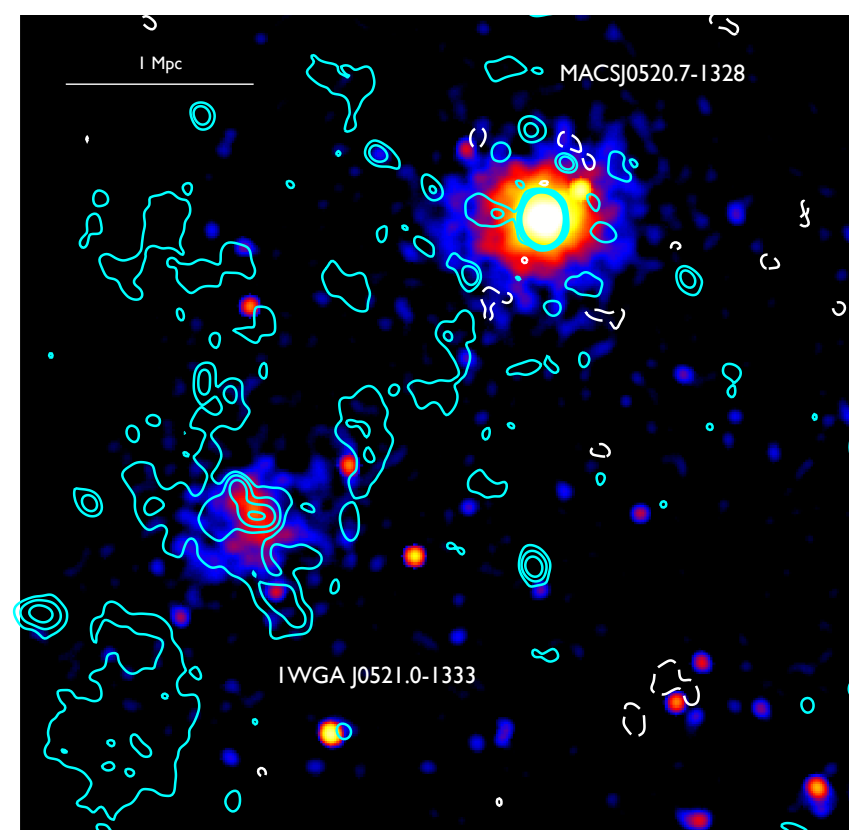

Fig. 3. Smoothed Chandra X-ray image in the $0.5-2.5 \mathrm{keV}$ energy band, with overlaid the $323 \mathrm{MHz}$ GMRT low-resolution contours (same as in Fig. 2, left panel; white are negative, cyan are positive).

the WGA Catalog (White et al. 1994). Compared to M 0520, its surface brightness distribution appears to be much less regular, with the brightest part of the X-ray emission slightly elongated in the NE-SW direction.

The image shown in Fig. 4 (left panel) is the result of a wavelet analysis including the denoising of the count rate map and the restoration of the X-ray surface brightness. More precisely, the count rate map has first been denoised from the $3 \sigma$ thresholding of a B3-spline undecimated wavelet transform. This was undertaken by assuming the variance of the wavelet coefficients to only be dependent on the effective exposure, thanks to the adaptation of a multiscale variance stabilization transform (see details in Zhang et al. 2008). The exposure-corrected background map has been further projected into the same significant multi-resolution support (Murtagh et al. 1995) and subtracted from the denoised count rate, yielding the X-ray surface brightness.

\subsection{Spectral analysis}

Spectral analyses were performed assuming a redshifted and $N_{\mathrm{H}}$ absorbed ICM emission spectrum modelled using the astrophysical plasma emission code (APEC, Smith et al. 2001), with the element abundances of Grevesse \& Sauval (1998) and neutral hydrogen absorption cross sections of BalucinskaChurch \& McCammon (1992). The $N_{\mathrm{H}}$ value was fixed to $7.3 \times$ $10^{24} \mathrm{~m}^{-2}$, from measurements obtained near M0520 in the Leiden/Argentine/Bonn Survey of galactic HI (Kalberla et al. 2005). ICM emission spectra were altered by the effective exposure and background noise, but also convolved by a redistribution function of the photon energies by the imaging camera. First tabulated within 128 tails of the ACIS-I CCDs using the Chandra Interactive Analysis of Observations (CIAO) software and CALDB 4.4.5, this function was computed for each spectrum by averaging the redistribution functions associated with each event position. 

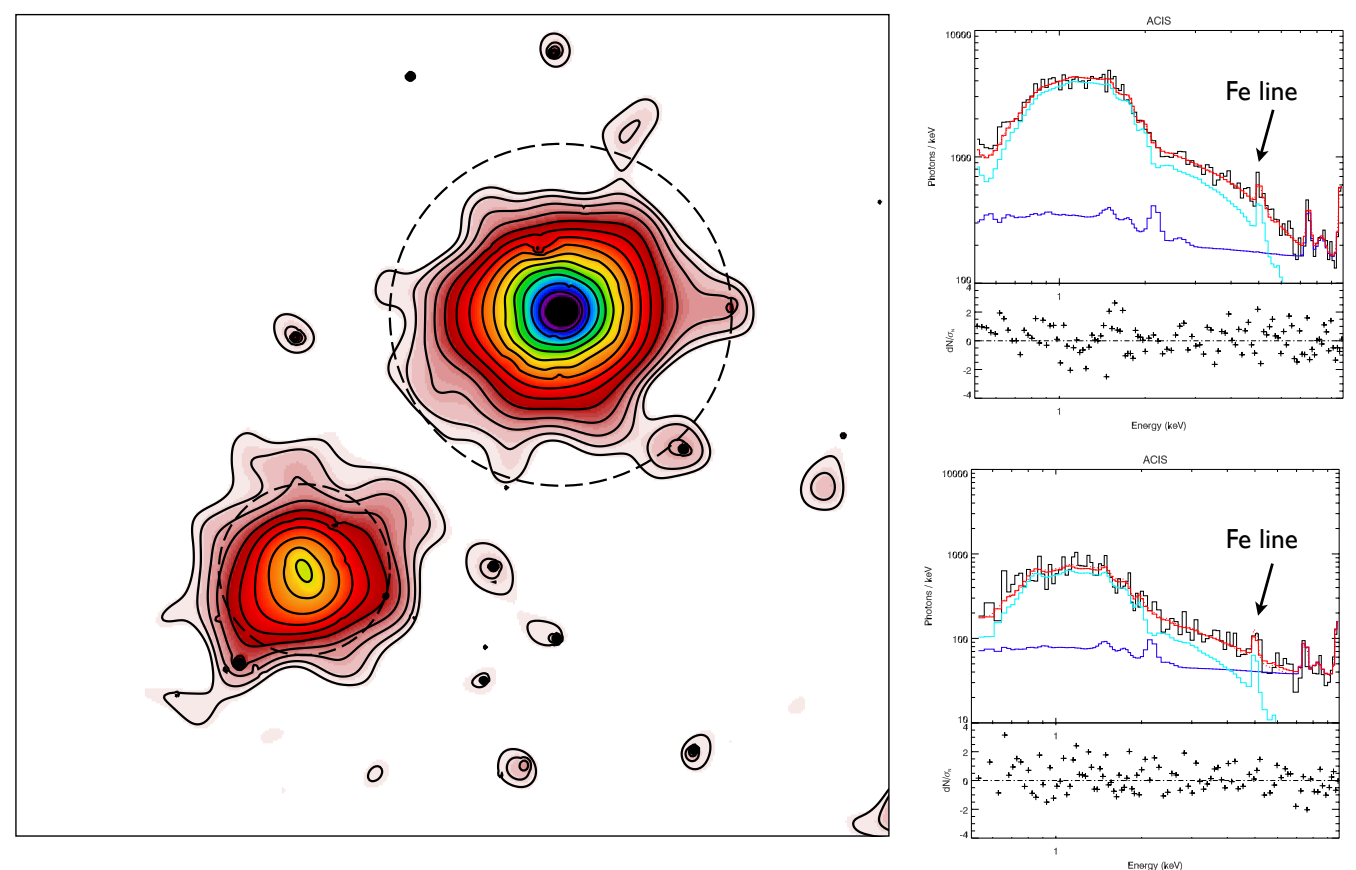

Fig. 4. Left panel: wavelet-reconstructed Chandra X-ray image in the $0.5-2.5 \mathrm{keV}$ energy band. The dashed circles correspond to $1 \mathrm{Mpc}$ for M 0520 and $0.5 \mathrm{Mpc}$ for 1WGA 0521, which have been used to extract the spectra. Right panel: X-ray spectra of the two extended sources (top: M 0520, bottom: 1WGA 0521), extracted from the event list in the $0.5-10 \mathrm{keV}$ band.

Table 2. X-ray properties of the two galaxy clusters.

\begin{tabular}{|c|c|c|c|c|c|c|c|c|}
\hline Cluster & $\begin{array}{l}\mathrm{RA}_{J 2000} \\
(\mathrm{~h}, \mathrm{~m}, \mathrm{~s})\end{array}$ & $\begin{array}{c}\operatorname{Dec}_{J 2000} \\
\left({ }^{\circ},,^{\prime},{ }^{\prime}\right)\end{array}$ & $z$ & $\begin{array}{c}k T \\
{[\mathrm{keV}]}\end{array}$ & $\begin{array}{c}Y_{X} \\
{\left[10^{14} M_{\odot} \mathrm{keV}\right]}\end{array}$ & $\begin{array}{c}M_{\mathrm{g}, 500} \\
{\left[10^{14} M_{\odot}\right]}\end{array}$ & $\begin{array}{c}M_{500} \\
{\left[10^{14} M_{\odot}\right]}\end{array}$ & $\begin{array}{c}r_{500} \\
{[\mathrm{kpc}]}\end{array}$ \\
\hline MACSJ 0520-1328 & 052042.0 & -132850 & $0.336_{-0.002}^{+0.004}$ & $6.2_{-1.2}^{+2.4}$ & $4.0_{-0.8}^{+1.6}$ & $0.65_{-0.05}^{+0.07}$ & $5.3_{-0.8}^{+1.5}$ & $1097_{-60}^{+93}$ \\
\hline 1WGA J0521.0-1333 & 052103.3 & -133405 & $0.34_{-0.02}^{+0.01}$ & $3.6_{-0.9}^{+1.4}$ & $1.1_{-0.3}^{+0.4}$ & $0.29_{-0.03}^{+0.04}$ & $2.5_{-0.4}^{+0.6}$ & $851_{-48}^{+63}$ \\
\hline
\end{tabular}

We have extracted the spectra of the two extended sources M 0520 and 1WGA 0521 from the event list in the $0.5-10 \mathrm{keV}$ band, within two point-source-excluded circular regions centred on each of them, with radii of $r=1 \mathrm{Mpc}$ and $r=0.5 \mathrm{Mpc}$, respectively. As shown in Fig. 4 (right panel), these spectra are ideally fit with two ICM emission spectra including their iron line complex redshifted near $5 \mathrm{keV}$. This identifies both sources as galaxy clusters. Our spectral fit allowed us to estimate two redshift values of $0.336_{-0.002}^{+0.004}$ and $0.34_{-0.02}^{+0.01}$ as for M 0520 and 1WGA 0521, respectively, consistent with each other and with the redshift of M0520 measured from optical spectroscopy (see also Table 2). The radii of the two regions used to extract our spectra have been empirically chosen in order to maximise the signal-to-noise ratio of the iron line, and thus best constrain these measurements. In the following estimates, we assume the redshift of both clusters to be fixed to this optical redshift, $z=0.336$. To estimate the cluster masses within $r_{500}$ - the radius of a sphere whose density is 500 times the critical density of the universe we computed their $Y_{X}$ parameter defined as the product of gas mass $M_{\mathrm{g}, 500}$ and average temperature $k T$ (Kravtsov et al. 2006). As described in, say, Bourdin \& Mazzotta (2008), we invert gas mass profiles from the radially average surface brightness of each cluster, then iterated about the $Y_{X}-M_{500}$ scaling relation calibrated from hydrostatic mass estimates in a nearby sample of clusters observed with Chandra (Vikhlinin et al. 2009). This yielded estimates of the total cluster masses $M_{500}$, radii $r_{500}$, gas masses $M_{\mathrm{g}, 500}$, and average temperatures $k T$, which are reported in Table 2.

\section{Optical/IR analysis}

In this section we investigate the optical/IR properties of the galaxy clusters M 0520 and 1 WGA 0521 by using available catalogues extracted from the WISE and UKST Red public surveys ${ }^{6}$.

\subsection{Colour-magnitude diagrams}

Galaxy clusters are characterised by a well defined sequence of red galaxies, most likely early-type cluster members (see e.g. Gladders \& Yee 2005). We used multi-band optical and infrared data to trace the colour-magnitude diagram (hereafter CMD) of galaxies in the central field on M0520 (see panel a of Fig. 5; $\sim 19^{\prime} \times 19^{\prime}$, same as in Fig. 1, left). For this, we matched the archival WISE and UKST Red catalogues sources (with a tolerance radius of $6^{\prime \prime}$ for association, i.e., $\sim 1 F W H M$ of the WISE band) and plotted the $R-3.4 \mu \mathrm{m}$ colour as a function of $\mathrm{R}$ magnitude. We selected only sources classified as galaxies based on their morphological parameters and areal profile shape ("CLASS $=1$ " sources in SuperCOSMOS catalog, Hambly et al. 2001). The CMD is shown in Fig. 5 (panels b and c). To trace it, we selected galaxies in the magnitude range $R=17.8-21$, i.e. within approximately the BCG magnitude and the UKST SES-R survey magnitude limit (Andernach 1999). Even though the field is dominated by cluster galaxies at quite high redshift

6 See http://irsa.ipac.caltech.edu/Missions/wise.html and http://www-wfau.roe.ac.uk/sss/index.html 

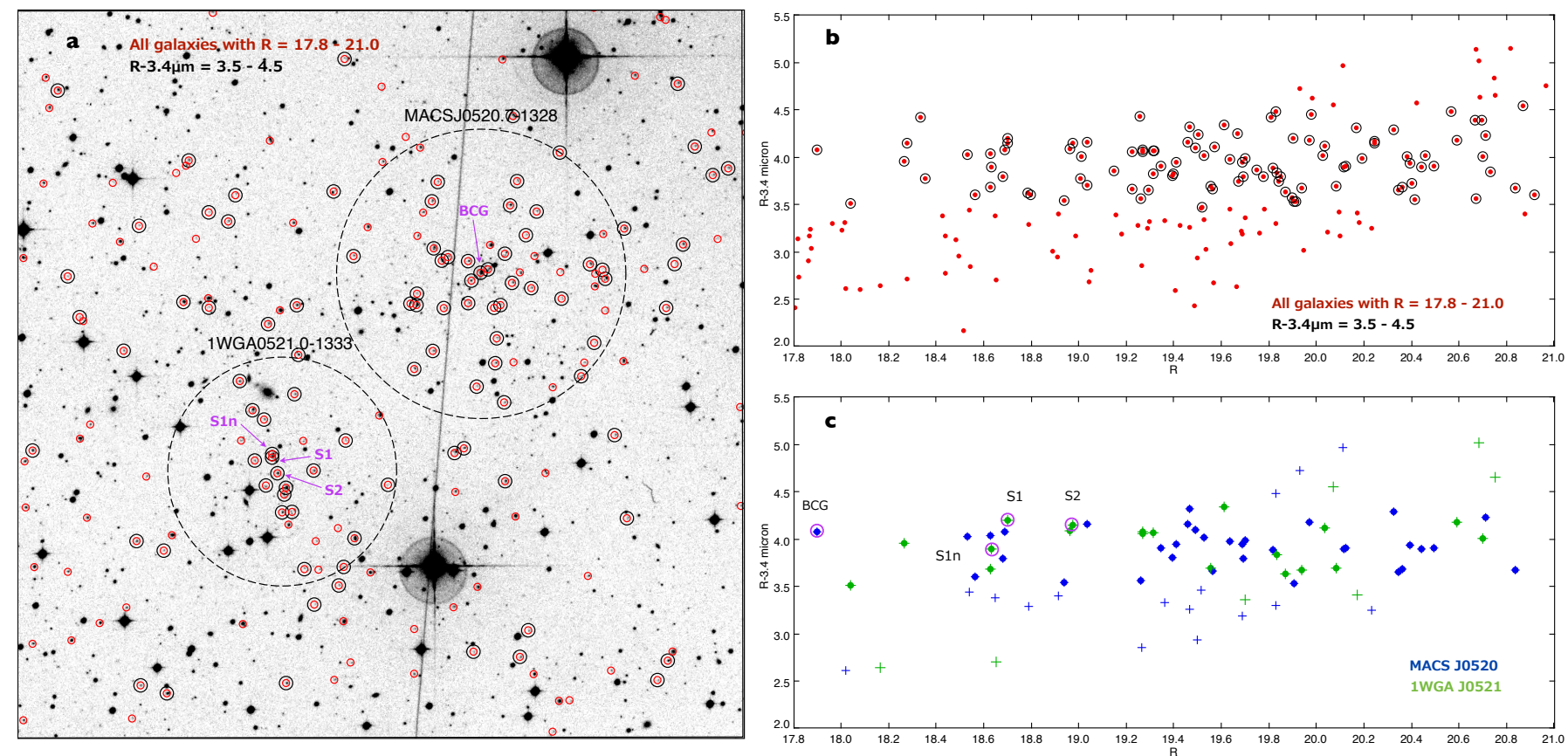

Fig. 5. a) DSS-R image of the $\sim 19^{\prime} \times 19^{\prime}$ area around M0520 (same as in Fig. 1, Right panel); dashed circles have radii $r_{500}$ and are centred on the X-ray centre of each cluster. The red small circles mark the galaxies selected in the range $R=17.8-21.0$; the large black circles show only those galaxies within the range of colours $R-3.4 \mu \mathrm{m}=3.5-4.5$. b) $R-3.4 \mu \mathrm{m}$ CMD of the galaxies within the $\sim 19^{\prime} \times 19^{\prime}$ area as in panel a) red and black circles as in panel a). c) $R-3.4 \mu \mathrm{m}$ CMD of the galaxies within the dashed circles in panel a) the crosses are all the galaxies in the range $R=17.8-21.0$, the filled dots are the colour-selected once $(R-3.4 \mu \mathrm{m}=3.5-4.5)$. The BCG of M 0520 and the optical counterparts of radio sources $\mathrm{S} 1$ and $\mathrm{S} 2$ are marked by magenta circles (they are also highlighted by magenta arrows in panel a), for clarity).

for the magnitude limit of SuperCOSMOS catalogue $(R * \sim 19.7$ at $z \sim 0.336)$, we identify the most likely red-sequence region in the CMDs, in the range of colours 3.5-4.5 (see Fig. 5, panel b).

Panel c of Fig. 5 shows the $R-3.4 \mu \mathrm{m}$ CMD for the galaxies located within circular areas of radii $1 r_{500}$ (see Table 2 ) from the X-ray centre of each cluster (see Fig. 5a). As is clear from Fig. 5 (panel a), almost all the galaxies within these two cluster regions fall in the colour range $\sim 3.5-4.5$, suggesting they all populate the most likely identified red sequence region.

In particular, most of the objects located in the 1WGA 0521 region (Fig. 5, panel c) populate the same locus of the CMD diagrams as the BCG of M0520 and as galaxies within the $\mathrm{r}_{500}$ radius of the main cluster (Fig. 5, panel c). This is especially true for the likely optical counterparts of the two radio galaxies S1 and S2 (see Fig. 1, right panel). Two galaxies actually lies within the $10 \sigma$ contour around the radio peak of S, labelled as S1n and S1 in (see Fig. 5, panel c); they both could be the optical counterpart of $\mathrm{S} 1$, and have similar colours.

We have statistically compared the distribution in the CMD of the galaxies lying within the central regions of the two clusters (i.e. the two circles shown in 5, panel a). According to a 2D Kolmogorov-Smirnov test and with a significance level $\lesssim 5 \%$, we can exclude that the two galaxy distributions are drawn from a different population.

\subsection{Galaxy isodensity map and multi-wavelength comparison}

Figure 6 shows the galaxy iso-density map in the same $19^{\prime} \times$ $19^{\prime}$ centred in between the clusters M0520 and 1WGA 0521. We have used the same catalogues as for the CMD analysis, selecting only galaxies in the $R$ magnitude range 17.8-21.0 and in the $3.4 \mu \mathrm{m}-R$ colour range $3.5-4.5$ (from UKST and WISE; see
Sect. 4.1). The map has been derived on the basis of a multi-scale approach, as described in Ferrari et al. (2005), and has been optimised to point out large-scale substructures. The optical isodensity contours are superposed on X-ray contours (same as in Fig. 4, left), and the $323 \mathrm{MHz}$ GMRT radio image (greyscale; from same image as in Fig. 2 left).

There is a very good match between the X-ray and optical emission of both clusters. M0520 appears to have a more regular, roundish shape (both in the X-ray and the optical), except in the central brightest X-ray region, where the iso-density contours are elongated towards NE. On the other hand, the projected galaxy distribution of 1WGA 0521 is far from being spherically symmetric with a clear elongation in the SW/NE direction, resembling the X-ray surface brightness distribution (see Fig. 3); in particular, most of the galaxies within its $r_{500}$ appear to be distributed along a very narrow line. Since it is expected that the gas and galaxies follow a similar distribution, these results support the idea that M0520 is a quite relaxed system, while the more irregular 1WGA 0521 is a disturbed system.

The diffuse radio source D1 is elongated in a similar direction to that of the main galaxy clump of $1 \mathrm{WGA} 0521$, and the two diffuse components D2 and D3 partially match the lowest optical iso-density contour of M 0520 . In contrast, D4 is located in a outer region, covering an area where no X-ray emission is detected or significant concentration of galaxies is present (at the level of the survey's magnitude limit and of the Chandra exposure).

\section{Discussion and conclusions}

We have reported high-sensitivity GMRT radio observations at $323 \mathrm{MHz}$ of the galaxy cluster M 0520, that allowed the detection of complex diffuse radio emission. The main feature of this emission is located at a projected distance of $\sim 8^{\prime}$ SE of 


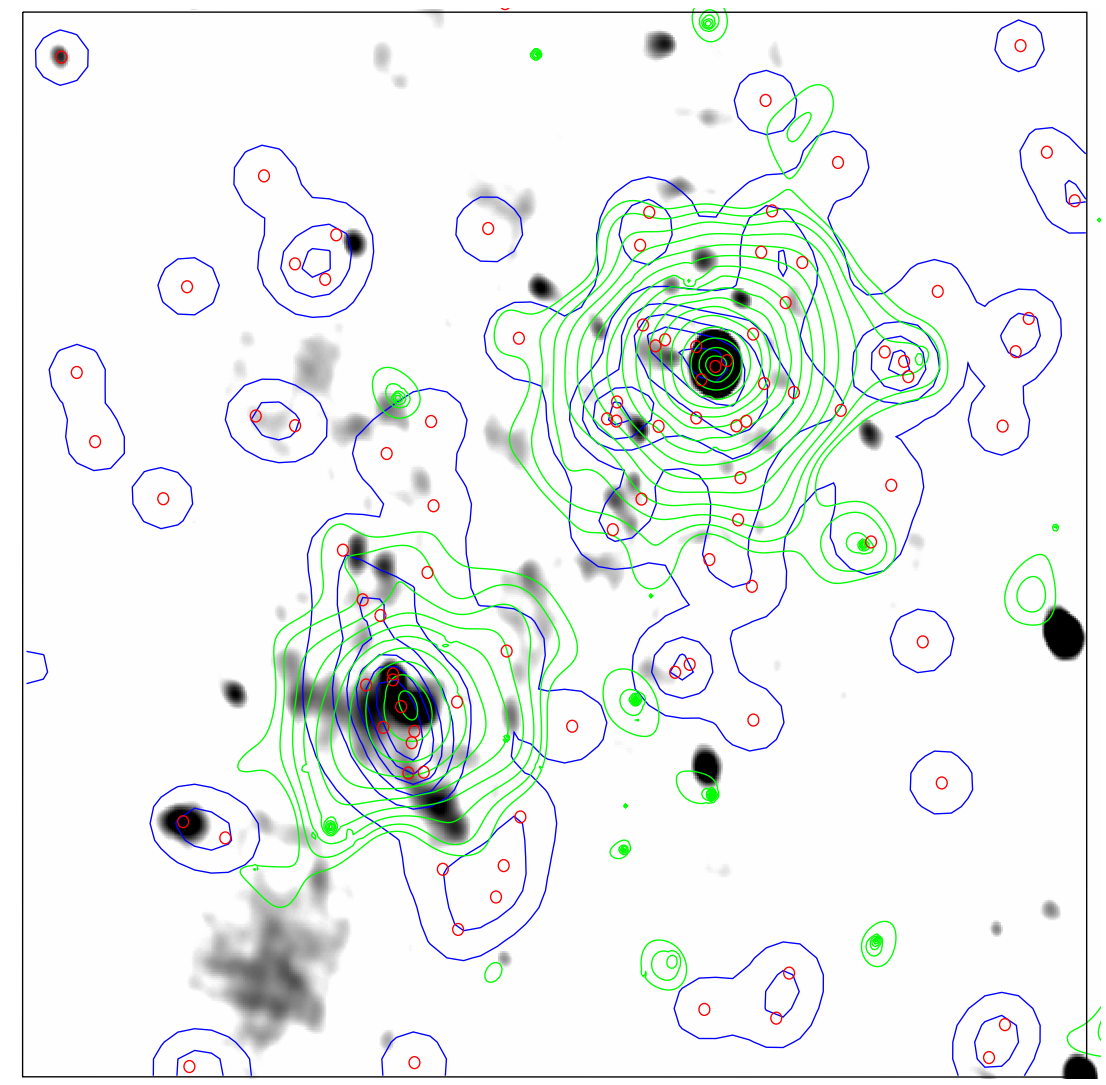

Fig. 6. Galaxy isodensity map (blue contours), with overlaid the X-ray Chandra contours (green, same as in Fig. 4). The radio image (same as in Fig. 2, left) is shown in greyscale (with the lowest level corresponding to $3 \sigma_{323 \mathrm{MHz}}=0.82 \mathrm{mJy} / \mathrm{b}$ ). Only galaxies in the magnitude range $R=$ $(17.8-21)$ and in the colour range $3.4 \mu \mathrm{m}-R=(3.5-4.5)$ have been used, and their spatial distribution is shown by red circles.

the cluster centre and was found to coincide with an extended $\mathrm{X}$-ray source seen in the archival Chandra image. We performed a multi wavelength analysis based on archival X-ray and optical/IR data, which allowed us to classify it as a galaxy cluster, namely 1 WGA 0521.

Through our X-ray analysis we characterised this newly discovered cluster as a low-temperature, relatively small system. From the spectral fitting we derived a redshift that is consistent with the optical redshift of M0520, $z=0.336$. However the uncertainties on our X-ray estimate (Sect. 3.2, Table 2) do not allow us to firmly conclude that the two clusters lie at the same distance $^{7}$. Even though at $z=0.336$ their projected separation of $\sim 2.3 \mathrm{Mpc}$ would easily include the clusters virial radii, the Chandra image does not show evidence of a bridge of X-ray emission between M0520 and 1WGA0521, which could be expected in case of interaction (though this may also be due to the low sensitivity of the exposure).

On the other hand, our optical/IR analysis supports the hypothesis that the two clusters lie at similar redshifts, since their galaxy populations lie in statistically similar regions of the colour-magnitude diagram. Optical spectroscopic observations and deeper X-ray observations are needed to accurately determine the 1WGA 0521 redshift and to investigate the dynamical scenario of these two systems and possible ongoing physical interaction.

\footnotetext{
7 At $z \sim 0.336$, the difference of 0.004 between the X-ray redshift estimates (see Table 2) corresponds to $25 \mathrm{Mpc}$ in luminosity distance. If we also consider their uncertainties from spectral fitting, the distance between the two clusters could be much greater.
}

The optical iso-density map derived from candidate cluster members shows that 1WGA 0521 has an elongated morphology, suggesting a disturbed dynamical state. Conversely, M 0520 seems more relaxed (as found by Ebeling et al. 2010), with a more regular shape, except for its inner region where the galaxy distribution is slightly elongated.

Our radio images at low resolution reveal a complex distribution of diffuse emission, which we have analysed by identifying four structures (namely D1, D2, D3, and D4, see Sect. 2). The diffuse elongated source D1 is the brightest of these features. It is characterised by large-scale and low-surface-brightness, and it is coincident with the newly detected, probably unrelaxed cluster of galaxy 1WGA 0521 . These properties are typical of clusterscale diffuse radio sources. Owing to its projected location in the central region of the cluster, the emission could be a cluster radio halo with an elongated morphology. Such a shape may also suggest the source is a radio relic (though located closer to the cluster centre with respect to what is typically observed). The emission in this case would be associated with the passage of a shock wave that may have either accelerated particles from the thermal ICM or revived fossil radio plasma from a previous activity of a radio-loud AGN (which could be one of the two nearby radio galaxies $\mathrm{S} 1$ and $\mathrm{S} 2$ ). Following the definition by Kempner et al. (2004), the source would be classified as radio gischt in the former scenario or a radio phoenix in the latter. The relic could be associated with the cluster M 520, rather than with 1WGA 0521. This hypothesis seems less likely, due to its large distance from the cluster centre $(\sim 2.3 \mathrm{Mpc})$, much greater than what is typically observed, and the fact that M 520 seems to be 
a relaxed system, while relics are always found to be associated to merging clusters. Another possibility that cannot be firmly excluded is that D1 is actually the tail of a tailed radio galaxy, whose nucleus may be identified with S1 or S2. Our present radio data, however, do not allow us to distinguish these possibilities, so spectral and polarisation information is needed to clearly classify this source.

Beyond the prominent source D1, additional diffuse emission on a large scale is located around it (D2, D3, D4). The two elongated structures D2 and D3 are difficult to classify. They have an even lower brightness, their shape is filamentary, and they are located in a region in between 1WGA0521 and M 0520. D4 is even harder to interpret, due to its roundish shape and its location in a region where our present data does not show any significant optical or X-ray emission. We suggest that these features might be related to non-thermal components in the faint inter-galactic medium surrounding 1WGA0521, which is not detected by the current shallow X-ray data owing to sensitivity limits, but may be revealed through more extensive observations (e.g. Planck Collaboration et al. 2013, and references therein).

Multi-frequency high-sensitivity radio observations are essential for properly classifying all the features of the complex extended radio emission and understanding their origins. Follow-up observations are planned.

Regardless of the nature of the diffuse radio emission and its possible relation to the cluster's dynamical state, the most important result of our analysis is that our detection of largescale diffuse radio emission has led us to discover a new massive galaxy cluster. Indeed, even though M0520 is a system in well studied cluster samples (Ebeling et al. 2010; Mantz et al. 2010; Horesh et al. 2010) and our X-ray and optical/IR analyses are based on archival data, the existence of the neighbouring cluster 1WGA0521 has been ignored up to now. Although it is quite common that X-ray surveys miss clusters (see e.g. Cagnoni et al. 2001), in this case the radio observations have been crucial to spotting its existence.

Thanks to ongoing and future deep all-sky radio surveys (e.g. LOFAR-Surveys, EMU-ASKAP, MeerKAT-MighTEE, MWA GLEAM), diffuse radio sources will become a powerful tool for discovering dynamically disturbed galaxy clusters and largescale structure merging/accretion events.

Acknowledgements. We thank the anonymous referee for useful comments. We are grateful to Gianfranco Brunetti and Monique Arnaud for helpful suggestions and fruitful discussions. We warmly thank Christophe Benoist for his help in deriving the galaxy isodensity map. We would like to thank the staff of the GMRT for making these observations possible. GMRT is run by the National Centre for Radio Astrophysics of the Tata Institute of Fundamental Research. The National Radio Astronomy Observatory is a facility of the National Science Foundation operated under cooperative agreement by Associated Universities, Inc. GM and CF acknowledge financial support by the "Agence Nationale de la Recherche" (ANR) through grant ANR-09-JCJC-0001-01. GWP acknowledges financial support by ANR through grant ANR-11-BD56-015.

\section{References}

Andernach, H. 1999, in Internet Resources for Professional Astronomy, eds. M. R. Kidger, I. Perez-Fournon, \& F. Sanchez (Cambridge University Press), 1

Balucinska-Church, M., \& McCammon, D. 1992, ApJ, 400, 699

Bartalucci, I., Mazzotta, P., Bourdin, H., \& Vikhlinin, A. 2014, A\&A, in press, DOI: $10.1051 / 0004-6361 / 201423443$

Blanton, E. L., Gregg, M. D., Helfand, D. J., Becker, R. H., \& White, R. L. 2003, AJ, 125, 1635

Booth, R. S., \& Jonas, J. L. 2012, African Skies, 16, 101

Bourdin, H., \& Mazzotta, P. 2008, A\&A, 479, 307

Cagnoni, I., Elvis, M., Kim, D.-W., et al. 2001, ApJ, 560, 86

Cassano, R., Brunetti, G., Röttgering, H. J. A., \& Brüggen, M. 2010a, A\&A, 509, A68

Cassano, R., Ettori, S., Giacintucci, S., et al. 2010b, ApJ, 721, L82

Cassano, R., Ettori, S., Brunetti, G., et al. 2013, ApJ, 777, 141

Chandra, P., Ray, A., \& Bhatnagar, S. 2004, ApJ, 612, 974

Condon, J. J., Cotton, W. D., Greisen, E. W., et al. 1998, AJ, 115, 1693

Ebeling, H., Edge, A. C., Mantz, A., et al. 2010, MNRAS, 407, 83

Enßlin, T. A., \& Röttgering, H. 2002, A\&A, 396, 83

Ferrari, C., Govoni, F., Schindler, S., Bykov, A. M., \& Rephaeli, Y. 2008, Space Sci. Rev., 134, 93

Feretti, L., Giovannini, G., Govoni, F., \& Murgia, M. 2012, A\&ARv, 20, 54

Galametz, A., Stern, D., Pentericci, L., et al. 2013, A\&A, 559, A2

Giacintucci, S., \& Venturi, T. 2009, A\&A, 505, 55

Gladders, M. D., \& Yee, H. K. C. 2005, ApJS, 157, 1

Grevesse, N., \& Sauval, A. J. 1998, Space Sci. Rev., 85, 161

Hambly, N. C., Irwin, M. J., \& MacGillivray, H. T. 2001, MNRAS, 326, 1295

Heald, G., et al. (LOFAR Collaboration) 2013, in AAS Meeting Abstracts, 221, 21507

Horesh, A., Maoz, D., Ebeling, H., Seidel, G., \& Bartelmann, M. 2010, MNRAS, 406, 1318

Intema, H. T., van der Tol, S., Cotton, W. D., et al. 2009, A\&A, 501, 1185

Johnston, S., Taylor, R., Bailes, M., et al. 2008, Exp. Astron., 22, 151

Kalberla, P. M. W., Burton, W. B., Hartmann, D., et al. 2005, A\&A, 440, 775

Kantharia, N. G., Das, M., \& Gopal-Krishna. 2009, J. Astrophys. Astron., 30, 37

Kassim, N. E., Lane, W. M., Cohen, A. S., et al. 2003, BAAS, 35, 1301

Kempner, J. C., Blanton, E. L., Clarke, T. E., et al. 2004, in The Riddle of Cooling Flows in Galaxies and Clusters of galaxies, eds. T. Reiprich, J. Kempner, \& N. Soker, 335

Kravtsov, A. V., Vikhlinin, A., \& Nagai, D. 2006, ApJ, 650, 128

Lane, W. M., Cohen, A. S., Cotton, W. D., et al. 2008, in Frontiers of Astrophysics: A Celebration of NRAO's 50th Anniversary, eds. A. H. Bridle, J. J. Condon, \& G. C. Hunt, ASP Conf. Ser., 395, 370

Lane, W. M., Cotton, W. D., Helmboldt, J. F., \& Kassim, N. E. 2012, Radio Sci., 47 [arXiv: 1205.4695]

Macario, G., Venturi, T., Intema, H. T., et al. 2013, A\&A, 551, A141

Mantz, A., Allen, S. W., Ebeling, H., Rapetti, D., \& Drlica-Wagner, A. 2010, MNRAS, 406, 1773

Mao, M. Y., Sharp, R., Saikia, D. J., et al. 2010, MNRAS, 406, 2578

Murtagh, F., Starck, J.-L., \& Bijaoui, A. 1995, A\&AS, 112, 179

Planck Collaboration 2013, A\&A, 550, A134

Planck Collaboration XXIX. 2014, A\&A, in press DOI:10.1051/0004-6361/201321523

Rengelink, R. B., Tang, Y., de Bruyn, A. G., et al. 1997, A\&AS, 124, 259

Smith, R. K., Brickhouse, N. S., Liedahl, D. A., \& Raymond, J. C. 2001, ApJ, 556, L91

Smolčić, V., Schinnerer, E., Finoguenov, A., et al. 2007, ApJS, 172, 295

Tingay, S. J., Goeke, R., Bowman, J. D., et al. 2013, PASA, 30, 7

van Haarlem, M. P., Wise, M. W., Gunst, A. W., et al. 2013, A\&A, 556, A2

Vikhlinin, A., Burenin, R. A., Ebeling, H., et al. 2009, ApJ, 692, 1033

White, N. E., Giommi, P., \& Angelini, L. 1994, BAAS, 26, 1372

Zhang, B., Fadili, M. J., Starck, J.-L., \& Digel, S. W. 2008, Stat. Methodol., 5, 387 